\title{
SIMPLE TEST FUNCTIONS IN MESHLESS LOCAL PETROV-GALERKIN METHODS
}

\author{
Ivatury S. Raju* \\ NASA Langley Research Center, Hampton, Virginia
}

\begin{abstract}
Two meshless local Petrov-Galerkin (MLPG) methods based on two different trial functions but that use a simple linear test function were developed for beam and column problems. These methods used generalized moving least squares (GMLS) and radial basis (RB) interpolation functions as trial functions. These two methods were tested on various patch test problems. Both methods passed the patch tests successfully. Then the methods were applied to various beam vibration problems and problems involving Euler and Beck's columns. Both methods yielded accurate solutions for all problems studied.

The simple linear test function offers considerable savings in computing efforts as the domain integrals involved in the weak form are avoided. The two methods based on this simple linear test function method produced accurate results for frequencies and buckling loads. Of the two methods studied, the method with radial basis trial functions is very attractive as the method is simple, accurate, and robust.
\end{abstract}

\section{Introduction}

In 1992, Nayroles et al. ${ }^{1}$ proposed a diffused element method based on moving least square approximating functions. Subsequent to this paper, considerable amount of research was invested in developing similar methods ${ }^{1-4}$. These diffused element methods came to be known as element-free, mesh-free, or meshless methods and are increasingly being viewed as an alternative to the finite element method. A meshless local Petrov-Galerkin (MLPG) formulation was introduced in reference 3. In the Galerkin formulations in references 2 and 4, the trial and test functions in the weak form come from the same space, while in the PetrovGalerkin $^{3}$ formulations, the trial and test funcions come from different spaces. In reference 4, a Galerkin formulation is presented for beam $\left(\mathrm{C}^{1}\right)$ problems using generalized moving least squares (GMLS) interpolants. In reference 5, a Petrov-Galerkin formulation is proposed with the GMLS interpolants as trial functions and spline functions as test functions. An alternative MLPG method with radial basis (RB) functions as trial functions that requires less computing effort is presented in reference 6. In this paper, MLPG methods with GMLS and RB as trial fuctions in association with a very simple test function (a linear function) are considered. These are referred to as the GMLS-Linear and RB-Linear MLPG methods. The performance of these methods is studied by applying these methods to vibrations of beams and buckling of Euler and Beck's columns.

\footnotetext{
* NASA Technical Fellow for Structures, NASA Engineering and Safety Center. Fellow AIAA, Member ASME, Member ASCE.
} 


\section{Analysis}

The governing equations of vibration of a beam and a column can be written as

$E I \frac{\partial^{4} W}{\partial x^{4}}+P \frac{\partial^{2} W}{\partial x^{2}}+\rho \frac{\partial^{2} W}{\partial t^{2}}=0$

where $E$ is the Young's modulus of the material, $I$ is the moment of inertia of the beam (or column), $W$ is the deflection at $(x, t), P$ is the compressive load applied to the column, $\rho$ is the mass density of the material, and $t$ denotes time. The solution to Eq. (1) is assumed to be

$W(x, t)=w(x) \cdot e^{i \omega t}$,

where $i=\sqrt{-1}$ and $\omega$ is the circular frequency. Substitution of Eq. (2) into Eq. (1) yields

$E I \frac{d^{4} w}{d x^{4}}+P \frac{d^{2} w}{d x^{2}}-\rho \omega^{2} w=0$

For beam problems, various boundary conditions are possible. For buckling of columns, the boundary conditons are such that one end is fixed at $x=0$ and a compressive load of $P$ is applied at $x=L$.

In the remainder of this section, first the development of the weak form of Eq. (3) is presented. Next, the trial and test functions are discussed. Then, the issues related to boundary conditions are addressed.

\section{A. Development of the Weak Form}

As an approximate solution to Eq. (3) is sought, a weak form of Eq. (3) is set up using a weight function $v(x)$ as

$$
\int_{\Omega}\left[E I \frac{d^{4} w}{d x^{4}}+P \frac{d^{2} w}{d x^{2}}-\rho \omega^{2} w\right] \cdot v(x) d x=0
$$

where $\Omega$ is the domain under consideration $(0 \leq x \leq L)$, see Figure 1. Integration of Eq. (4) twice by parts leads to 


$$
\begin{aligned}
& \int_{\Omega}\left[\frac{d^{2} v}{d x^{2}} \cdot E I \frac{d^{2} w}{d x^{2}}\right] d x-\left[n_{x} \frac{d v}{d x} \cdot E I \frac{d^{2} w}{d x^{2}}\right]_{\Gamma}+\left[n_{x} v \cdot E I \frac{d^{3} w}{d x^{3}}\right]_{\Gamma}+\int_{\Omega} v \cdot P \frac{d^{2} w}{d x^{2}} d x \\
& -\int_{\Omega} v \cdot \rho \omega^{2} w d x=0,
\end{aligned}
$$

where $\Gamma$ is the boundary of the domain $(x=0$ or $x=L)$, and $n_{x}$ is the direction cosine of the normal to the boundary $\left(n_{x}=1\right.$ when $x=L$, and $n_{x}=-1$ when $x=0$ ). Sometimes a penalty method is used to prescribe essential boundary conditions. In such situations, Eq. (5) includes the penalty terms, and the weak form appears as

$$
\begin{aligned}
& \int_{\Omega}\left[\frac{d^{2} v}{d x^{2}} \cdot E I \frac{d^{2} w}{d x^{2}}\right] d x-\left[n_{x} \frac{d v}{d x} \cdot E I \frac{d^{2} w}{d x^{2}}\right]_{\Gamma}+\left[n_{x} v \cdot E I \frac{d^{3} w}{d x^{3}}\right]_{\Gamma}+\int_{\Omega} v \cdot P \frac{d^{2} w}{d x^{2}} d x-\int_{\Omega} v \cdot \rho \omega^{2} w d x \\
& +\alpha_{w}[(w-\widetilde{w}) \cdot v]_{\Gamma_{w}}+\alpha_{\theta}\left[\left(\frac{d w}{d x}-\widetilde{\theta}\right) \cdot \frac{d v}{d x}\right]_{\Gamma_{\theta}}=0
\end{aligned}
$$

Consider a beam and its $N$-node meshless model as shown in Figure 1. In the MLPG methods, the trial functions are assumed at each of the nodes in the model (and are diffused unlike in the finite element method), and the test functions are assumed independently (from a different space compared to the trial functions) as ${ }^{5}$

$w(x)=\sum_{j=1}^{N}\left(w_{j} \psi_{j}^{(w)}(x)+\theta_{j} \psi_{j}^{(\theta)}(x)\right)$

and

$$
v(x)=\mu_{i}^{(w)} \chi_{i}^{(w)}(x)+\mu_{i}^{(\theta)} \chi_{i}^{(\theta)}(x),
$$

where $w_{j}$ and $\theta_{j}$ are the nodal values of the deflection and slope at node $j$, and $\psi_{j}^{(w)}$ and $\psi_{j}^{(\theta)}$ are the assumed trial functions for deflection and slope, respectively. Similarly, the $\chi_{i}^{(w)}$ and $\chi_{i}^{(\theta)}$ are the assumed test functions and $\mu_{i}^{(w)}$ and $\mu_{i}^{\left({ }^{(\partial)}\right.}$ are arbitrary constants.

A typical trial function at node $j$ and test function at node $i$ are shown in Figure 2 . The test functions can be conveniently chosen to be nonzero only in the neighborhood of node $i$ ( $\Omega_{S}^{i} ; x_{i}-R_{0}$ to $x_{i}+R_{0}$ ), where $R_{0}$ is a user defined parameter and $\Omega_{S}$ describes the extent of the test function. As mentioned previously, the trail function is diffused and the extent of the trial function is as denoted by $x_{j}-R_{j}$ to $x_{j}+R_{j}$. A typical trial function at node $j$ and test function at node $i$ are shown in Figure 2. With these assumptions, the weak form of Eq. (6) reduces to 


$$
\begin{aligned}
& \int_{\Omega_{s}^{i}}\left[\frac{d^{2} v}{d x^{2}} \cdot E I \frac{d^{2} w}{d x^{2}}\right] d x-\left[n_{x} \frac{d v}{d x} \cdot E I \frac{d^{2} w}{d x^{2}}\right]_{\Gamma^{i} s}+\left[n_{x} v \cdot E I \frac{d^{3} w}{d x^{3}}\right]_{\Gamma_{s}^{i}}+\int_{\Omega_{s}^{i}} v \cdot P \frac{d^{2} w}{d x^{2}} d x-\int_{\Omega_{s}^{i}} v \cdot \rho \omega^{2} w d x \\
& +\alpha_{w}[(w-\widetilde{w}) \cdot v]_{\Gamma_{w}}+\alpha_{\theta}\left[\left(\frac{d w}{d x}-\widetilde{\theta}\right) \cdot \frac{d v}{d x}\right]_{\Gamma_{\theta}}=0
\end{aligned}
$$

where $\Gamma_{s}^{i}$ are the two ends of the domain $\Omega_{S}^{i}$. Substitution of the trial and test functions into the weak form and requiring that the weak form be valid for arbitrary values of $\mu_{i}^{(w)}$ and $\mu_{i}^{(\theta)}$ leads to

$[K]^{\text {node }}\{d\}+[K]^{\text {bdry }}\{d\}+P\left[K_{G}\right]\{d\}-\rho \omega^{2}[M]\{d\}=\{0\}$,

where

$$
\begin{aligned}
& \{d\}=\left\{\begin{array}{lllllllll}
w_{1} & \theta_{1} & w_{2} & \theta_{2} & w_{3} & \theta_{3} & \ldots \ldots & \ldots & \ldots \\
w_{N} & \theta_{N}
\end{array}\right\} \\
& \mathbf{k}_{i j}^{\text {(node) }}=E I\left[\begin{array}{cc}
\int_{\Omega_{s}^{(i)}} \frac{d^{2} \chi_{i}^{(w)}}{d x^{2}} \frac{d^{2} \psi_{j}^{(w)}}{d x^{2}} d x & \int_{\Omega_{s}^{(i)}} \frac{d^{2} \chi_{i}^{(w)}}{d x^{2}} \frac{d^{2} \psi_{j}^{(\theta)}}{d x^{2}} d x \\
\int_{\Omega_{s}^{(i)}} \frac{d^{2} \chi_{i}^{(\theta)}}{d x^{2}} \frac{d^{2} \psi_{j}^{(w)}}{d x^{2}} d x & \int_{\Omega_{s}^{(i)}} \frac{d^{2} \chi_{i}^{(\theta)}}{d x^{2}} \frac{d^{2} \psi_{j}^{(\theta)}}{d x^{2}} d x
\end{array}\right] \\
& +n_{x} E I\left[\begin{array}{cc}
\chi_{i}^{(w)} \frac{d^{3} \psi_{j}^{(w)}}{d x^{3}} & \chi_{i}^{(w)} \frac{d^{3} \psi_{j}^{(\theta)}}{d x^{3}} \\
\chi_{i}^{(\theta)} \frac{d^{3} \psi_{j}^{(w)}}{d x^{3}} & \chi_{i}^{(\theta)} \frac{d^{3} \psi_{j}^{(\theta)}}{d x^{3}}
\end{array}\right]_{\Gamma_{s I}^{(i)}} \\
& -n_{x} E I\left[\begin{array}{cc}
\frac{d \chi_{i}^{(w)}}{d x} \frac{d^{2} \psi_{j}^{(w)}}{d x^{2}} & \frac{d \chi_{i}^{(w)}}{d x} \frac{d^{2} \psi_{j}^{(\theta)}}{d x^{2}} \\
\frac{d \chi_{i}^{(\theta)}}{d x} \frac{d^{2} \psi_{j}^{(w)}}{d x^{2}} & \frac{d \chi_{i}^{(\theta)}}{d x} \frac{d^{2} \psi_{j}^{(\theta)}}{d x^{2}}
\end{array}\right]_{\Gamma_{s I}^{(i)}}
\end{aligned}
$$




$$
\begin{aligned}
& \mathbf{k}_{i j}^{(\mathrm{bdry})}=\alpha_{w}\left[\begin{array}{cc}
\chi_{i}^{(w)} \psi_{j}^{(w)} & \chi_{i}^{(w)} \psi_{j}^{(\theta)} \\
\chi_{i}^{(\theta)} \psi_{j}^{(w)} & \chi_{i}^{(\theta)} \psi_{j}^{(\theta)}
\end{array}\right]_{\Gamma_{s w}^{(i)}} \\
& +n_{x} E I\left[\begin{array}{cc}
\chi_{i}^{(w)} \frac{d^{3} \psi_{j}^{(w)}}{d x^{3}} & \chi_{i}^{(w)} \frac{d^{3} \psi_{j}^{(\theta)}}{d x^{3}} \\
\chi_{i}^{(\theta)} \frac{d^{3} \psi_{j}^{(w)}}{d x^{3}} & \chi_{i}^{(\theta)} \frac{d^{3} \psi_{j}^{(\theta)}}{d x^{3}}
\end{array}\right]_{\Gamma_{s w}^{(i)}} \\
& +\alpha_{\theta}\left[\begin{array}{cc}
\frac{d \chi_{i}^{(w)}}{d x} \frac{d \psi_{j}^{(w)}}{d x} & \frac{d \chi_{i}^{(w)}}{d x} \frac{d \psi_{j}^{(\theta)}}{d x} \\
\frac{d \chi_{i}^{(\theta)}}{d x} \frac{d \psi_{j}^{(w)}}{d x} & \frac{d \chi_{i}^{(\theta)}}{d x} \frac{d \psi_{j}^{(\theta)}}{d x}
\end{array}\right]_{\Gamma_{s \theta}^{(i)}}, \\
& -n_{x} E I\left[\begin{array}{cc}
\frac{d \chi_{i}^{(w)}}{d x} \frac{d^{2} \psi_{j}^{(w)}}{d x^{2}} & \frac{d \chi_{i}^{(w)}}{d x} \frac{d^{2} \psi_{j}^{(\theta)}}{d x^{2}} \\
\frac{d \chi_{i}^{(\theta)}}{d x} \frac{d^{2} \psi_{j}^{(w)}}{d x^{2}} & \frac{d \chi_{i}^{(\theta)}}{d x} \frac{d^{2} \psi_{j}^{(\theta)}}{d x^{2}}
\end{array}\right]_{\Gamma_{\mathrm{s} \theta}^{(i)}} \\
& {\left[\mathbf{K}_{G}\right]=\left[\begin{array}{cc}
\int_{\Omega_{s}^{i}} \chi_{i}^{(w)} \frac{d^{2} \psi_{j}^{(w)}}{d x^{2}} d x & \int_{\Omega_{s}^{i}} \chi_{i}^{(w)} \frac{d^{2} \psi_{j}^{(\theta)}}{d x^{2}} d x \\
\int_{\Omega_{s}^{i}} \chi_{i}^{(\theta)} \frac{d^{2} \psi_{j}^{(w)}}{d x^{2}} d x & \int_{\Omega_{s}^{i}} \chi_{i}^{(\theta)} \frac{d^{2} \psi_{j}^{(\theta)}}{d x^{2}} d x
\end{array}\right],}
\end{aligned}
$$

and

$$
[\mathbf{M}]=\left[\begin{array}{ll}
\int_{\Omega_{i}^{i}} \chi_{i}^{(w)} \psi_{j}^{(w)} d x & \int_{\Omega_{s}^{i}} \chi_{i}^{(w)} \psi_{j}^{(\theta)} d x \\
\int_{\Omega_{s}^{i}} \chi_{i}^{(\theta)} \psi_{j}^{(w)} d x & \int_{\Omega_{s}^{i}} \chi_{i}^{(\theta)} \psi_{j}^{(\theta)} d x
\end{array}\right]
$$


The variables $\alpha_{w}$ and $\alpha_{\theta}$ in Eqs. (9) and (12) are penalty parameters used to prescribe the boundary conditions ${ }^{3-5}$. The $\Gamma_{s w}$ and $\Gamma_{s \theta}$ are the parts of the boundaries where $w$ and $\theta$ are prescibed.

The eigenvalue equation of Eq.(10) can be rewritten a

$$
\rho \omega^{2}\{d\}=[M]^{-1} \cdot\left([K]+P \cdot\left[K_{G}\right]\right) \cdot\{d\},
$$

where $[M]$ is the 'mass' matrix, $[K]$ is the 'stiffness' matrix that includes the contributions of $[K]^{\text {node }}$ and $[K]^{\text {bdry }}$, and $\left[K_{G}\right]$ is the 'geometric stiffness' matrix that may include the nonconservative contributions of the load. For free vibrations, $P$ is set equal to zero in Eq. (15). For buckling problems, the critical buckling load is evaluated by setting $\omega=0$ in Eq. (15). Note that the vector $\{d\}$ in Eq. (15) is the vector of fictitious nodal values $\{\hat{d}\}$ for the GMLS models, while $\{\tilde{d}\}$ is the vector of actual nodal values for the RB models.

\section{B. Trial and Test Functions}

Two different trial functions for $w$, GMLS and RB functions, are chosen in the weak form to develop the GMLS and RB meshless methods. The GMLS trial functions are discussed in references 4 and 5 , the RB trial functions in reference 6 , and hence are not repeated here. In this paper, the GMLS trial functions use quartic basis functions (see Ref. 5), while the RB uses cubic basis (see Ref. 6).

The GMLS trial functions are based on the moving least square (MLS) approximation ${ }^{1,3-5}$. The MLS approximation does not possess the Kronecker delta property as it uses fictitious nodal values and least squares fit to these nodal values to define the MLS approximation. A schematic of this fit is shown in Figure 3. This creates problems with the prescripition of the boundary conditions. (The issues related to the boundary condition presciption are discussed in a later section.) In contrast, the radial basis trial functions pass through the nodal values (hence possess Kronecker delta property) and hence the boundary conditions are easier to prescribe.

For both GMLS and RB methods, simple linear test functions are chosen. A typical test function at node $i$ is shown in Figure 4,

$$
\begin{array}{ll}
\chi_{i}^{(w)}(x)=\left(x-x_{i}\right) & \text { if } 0 \leq \delta_{i} \leq R_{o}, \text { where } \delta_{i}=\sqrt{\left(x-x_{i}\right)^{2}} \\
\chi_{i}^{(\theta)}(x)=1 &
\end{array}
$$

and 
$\chi_{i}^{(w)}(x)=0$

$\chi_{i}^{(\theta)}(x)=0$

if $\delta_{i}>R_{o}$

so that

$\frac{d^{2} \chi_{i}^{(w)}}{d x^{2}}=\frac{d^{2} \chi_{i}^{(\theta)}}{d x^{2}}=0$

With this choice, the second derivatives of the test function are identically zero, and this leads to a null matrix for the first term in the $\left[K^{\text {node }}\right]$ matrix (see Eq. (10)). As such, the integrations over $\Omega_{s}$ are not needed. This results in significant saving in computing effort.

\section{Boundary Conditions}

For static problems, the penalty method is used to prescribe the boundary conditions. The variables, $\alpha_{w}$ and $\alpha_{\theta}$, in Eqs. (9) and (12) are the penalty parameters and are usually chosen to be $10^{10}$ times the $E I$ of the beam or the column.

For dynamic problems, the essential boundary conditions (EBCs) are homogeneous. Before Eq. (15) can be used to evaluate the eigenvlaues, the boundary conditions need to be used to arrive at the reduced stiffness and mass matrices. In the RB-linear method, the reduced stiffness and mass matrices can be obtained by deleting the row and columns that correspond to the EBCs in a manner similar to the finite element method ${ }^{7,8}$. As the fictitious nodal values of the GMLS approximations do not pass through the nodal values, the prescription of the EBCs is not straightforward. To overcome this problem, Ref. 9 suggested a simple method to relate the actual nodal values at node $j, \tilde{d}_{j}$, to the fictitious nodal values $\hat{d}_{j}$ using the GMLS shape functions. Similar relationships can be established for the test functions. These relationships can be expressed as

$\{\tilde{d}\}=[R]\{\hat{d}\} ; \quad\{\tilde{v}\}=[T]\{\hat{v}\}$

Using these two transformations, the reduced stiffness, mass, and geometric stiffness matrices can be obtained as

$$
\begin{aligned}
& {[\tilde{K}]=\left[T^{-1}\right]^{T}[K]\left[R^{-1}\right],} \\
& {[\tilde{M}]=\left[T^{-1}\right]^{T}[M]\left[R^{-1}\right],} \\
& \text { and } \\
& {[\tilde{G}]=\left[T^{-1}\right]^{T}[G]\left[R^{-1}\right]}
\end{aligned}
$$


The boundary conditions are then prescribed and the reduced matrices are evaluated in a manner similar to the finite element method.

Beams: The essential boundary conditions for a cantilever beam are $w=0$ and $(d w / d x)=$ $\theta=0$ at $x=0$. At $x=L$, the natural boundary conditions are

$$
E I \frac{d^{2} w}{d x^{2}}=E I \frac{d^{3} w}{d x^{3}}=0
$$

For pin-ended beams,

$$
\begin{aligned}
& w=0 \text { and } E I \frac{d^{2} w}{d x^{2}}=0 \\
& \text { at } x=0 \text { and } x=L .
\end{aligned}
$$

Columns: The essential boundary conditions $w=0$ and $(d w / d x)=\theta=0$ are prescribed at $x=0$, the fixed end of the column. At $x=L$, the loaded end of the column, the boundary conditions are natural and are defined as

$$
E I \frac{d^{2} w}{d x^{2}}=0 \text { and } E I \frac{d^{3} w}{d x^{3}}=-P \cdot(1-\gamma) \cdot \frac{d w}{d x}
$$

The shear force at $x=L$ depends on the orientation, $\gamma$, of the load, $P$. For the case of Euler buckling, the direction of the load does not change as the column buckles, i.e., $\gamma=0$.

The boundary conditions in Eq. (23) are implemented in the weak form by considering the $\Gamma_{s V}$ and $\Gamma_{s M}$ terms (i.e. the terms that correspond to the $\Gamma_{s i}$ when $x=L$ ) as

$$
-\left[n_{x} \frac{d v}{d x} \cdot E I \frac{d^{2} w}{d x^{2}}\right]_{\Gamma_{s M}}+\left[n_{x} v \cdot E I \frac{d^{3} w}{d x^{3}}\right]_{\Gamma_{s V}}
$$

At $x=L$, with $n_{x}=1$, one obtains these terms as

$$
\begin{aligned}
& =-0+\left[v \cdot\left\{-P \cdot(1-\gamma) \frac{d w}{d x}\right\}\right]_{x=L} \\
& =v \cdot\left\{-P \cdot(1-\gamma) \theta_{N}\right\}
\end{aligned}
$$

where $\gamma$ is the orientation of the load (see Figure 5). For an Euler column $\gamma=0$ and for Beck's column $\gamma=1^{10}$. When the load follows the column as it buckles, the load is tangential to the deformed position of the column. The follower force problem is 
nonlinear because of this boundary condition and hence $P$ is considered an nonconservative force ${ }^{10-12}$.

The terms in Eq. (25) are evaluated, and appropriate entries are made into the $[K]^{\text {bdry }}$ matrix.

\section{Numerical Evaluations}

In this section, the GMLS-Linear and RB-Linear methods are evaluated by applying them to various patch tests, vibration problems for beams and buckling problems for columns for which exact or very accurate solutions are available. Both the methods are then applied to the follower force problem to demonstrate their effectiveness.

A beam and a column of constant cross section $A$, density $\rho$, flexural rigidity $E I$ and a length of $L=4 l$ are considered. The length $l$ was specifically chosen to avoid scaling by a unit length. Four models with 5, 9, 17, and 33 nodes uniformly distributed along the length of the beam and column were considered. Figure 2 shows a typical 9-node model. The distance between the nodes $(\Delta x / l)$ in these models are 1, 0.5, 0.25, and 0.125 for the 5-, 9-, 17-, and 33-node models, respectively. The value of $\left(R_{o} / l\right)$ was chosen to be $(\{\Delta x / l\} / 2)$. The value of $\left(R_{j} / l\right)$ was set equal to $L$ for RB-Linear and $\left(R_{j} / l\right)$ was set equal to $4(\Delta x / l)$ for GMLS-Linear cases. Note that the $\left(R_{j} / l\right)$ defines the extent of the trial function i.e. it defines how diffused the trial function is.

\section{A. Patch Tests}

The GMLS-Linear and RB-Linear methods were applied to four simple patch-test problems. The problems considered were:

(a) Rigid body translation: $w(x)=\beta_{0} ; \theta=\frac{d w}{d x}=0$,

(b) Rigid body rotation: $w(x)=\beta_{1} x ; \theta=\beta_{1}$,

(c) Constant-curvature condition: $w(x)=\beta_{2}\left(x^{2} / 2\right) ; \theta=\beta_{2} x$,

and (d) linear curvature condition: $w(x)=\beta_{3} x^{3} ; \theta=3 \beta_{3} x^{2}$

where $\beta_{0}, \beta_{1}, \beta_{2}$, and $\beta_{3}$ are arbitrary constants. Note that these four problems represent $w=$ constant, linear, quadratic, and cubic conditions, respectively, in the beam. The third patch test represents the problem of a cantilever beam with an end moment $\mathrm{M}$ applied at $x=L$ :

$M=E I \frac{d^{2} w}{d x^{2}}=E I \beta_{2}$.

The deflection $w$, and the slope, $\theta$, for all the problems were evaluated at $x=0$ and $x=L$ and are prescibed as EBCs. The penalty method was used to prescribe the EBCs. All beam problems were analyzed with each of the four discritizations (5-, 9-, 17-, and 33-node models) using the GMLS-Linear and RB-Linear methods. Every case considered using both methods matched the 
exact solution for deflections, slopes, moments, and shear forces to within the machine accuracy in the entire beam domain. Thus both GMLS-Linear and RB-Linear models passed the patch tests successfully.

\section{B. Vibrations:}

Free vibrations of a beam with various boundary conditions were performed by setting $P=0$ in Eq. (15). The non-dimensional fundamental frequencies, $\left(\rho A \omega^{2} L^{4} / E I\right)$, for four modes of a free-free beam obtained with the four models (5-, 9-, 17-, and 33-node models) are presented in Table 1. Both methods recovered the first two rigid body mode frequencies to machine accuracy. The frequencies for the next two modes agree extremely well with the accurate solution from reference 14. Similarly, the non-dimensional fundamental frequencies, $\left(\rho A \omega^{2} L^{4} / E I\right)$, two modes of a fixed-free beam are presented in Table 2. All models gave accurate results; the results deviated by less than one percent from analytical solutions from reference 14.

Table 3 presents non-dimensional fundamental frequencies, $\left(\rho A \omega^{2} L^{4} / E I\right)$, for the first two modes for beams with various boundary conditions. These results are obtained with the 17node model. The computed frequencies by both methods agree extremely well with the accurate analytical results from reference 14 .

From numerical experimentation on the patch tests and the vibration problems, accurate results are obtained when the extent of the trail function was equal to the domain length for the RB method, while the GMLS method does not perform well when the extent is as large as the domain length. The values of $\left(R_{j} / l\right)=L$ for RB-Linear and $\left(R_{j} / l\right)=4(\Delta x / l)$ for GMLS-Linear cases provided accurate results.

All the eigenvlaues of the reduced order matrices are computed using the routines provided in Section 11.5 of Reference 15. In all the computations above, the RB-linear method yielded accurate eigenvalues and all the eigenvalues are always positive and real. The GMLS-Linear method sometimes yielded non zero and complex eigenvalues for extreme higher order modes, while lower order modes were positive and real. These results suggest that the GMLS-Linear method with the procedure outlined in Eqs. 19 and 20 may not be rigorous and needs further study.

Table 1: Non-dimensional frequencies $\left(\rho A \omega^{2} L^{4} / E I\right)$ for a free-free beam 


\begin{tabular}{|c|c|c|c|c|c|c|c|c|}
\hline \multirow{3}{*}{$\begin{array}{l}\text { N-Node } \\
\text { Model }\end{array}$} & \multicolumn{4}{|c|}{ GMLS-Linear } & \multicolumn{4}{|c|}{ RB-Linear } \\
\hline & \multicolumn{4}{|c|}{ Modes } & \multicolumn{4}{|c|}{ Modes } \\
\hline & 1 & 2 & 3 & 4 & 1 & 2 & 3 & 4 \\
\hline 5 & $E-10$ & E-11 & 499.7 & 3679 & E-13 & E-13 & 486.3 & 3454 \\
\hline 9 & $E-10$ & $\mathrm{E}-10$ & 499.2 & 3960 & E-11 & $\mathrm{E}-12$ & 496.7 & 3696 \\
\hline 17 & $\mathrm{E}-8$ & E-9 & 496.9 & 3809 & $\mathrm{E}-10$ & $\mathrm{E}-12$ & 499.5 & 3784 \\
\hline 33 & $E-8$ & $E-8$ & 501.5 & 3822 & $E-9$ & $E-11$ & 500.2 & 3796 \\
\hline Ref. 14 & 0 & 0 & 500.5 & 3804 & 0 & 0 & 500.5 & 3804 \\
\hline
\end{tabular}

Table 2: Non-dimensional frequencies $\left(\rho A \omega^{2} L^{4} / E I\right)$ for a fixed-free beam

\begin{tabular}{|c|c|c|c|c|}
\hline \multirow{3}{*}{$\begin{array}{c}\text { N-Node } \\
\text { Model }\end{array}$} & \multicolumn{2}{|c|}{ GMLS-Linear } & \multicolumn{2}{|c|}{ RB-Linear } \\
\hline & \multicolumn{2}{|c|}{ Modes } & \multicolumn{2}{|c|}{ Modes } \\
\hline & 1 & 2 & 1 & 2 \\
\hline 5 & 12.36 & 495.1 & 12.39 & 470.6 \\
\hline 9 & 12.36 & 478.7 & 12.36 & 481.5 \\
\hline 17 & 12.36 & 482.8 & 12.36 & 484.4 \\
\hline 33 & 12.36 & 486.4 & 12.36 & 485.4 \\
\hline Ref. 14 & 12.36 & 485.5 & 12.36 & 485.5 \\
\hline
\end{tabular}


Table 3: Non-dimensional frequencies $\left(\rho A \omega^{2} L^{4} / E I\right)$ for beams with various boundary conditions - 17-node model.

\begin{tabular}{|c|c|c|c|c|c|c|}
\hline & \multicolumn{2}{|c|}{ Fixed-Free } & \multicolumn{2}{c|}{ Fixed-Fixed } & \multicolumn{2}{c|}{ Pinned-Pinned } \\
\cline { 2 - 7 } & \multicolumn{2}{|c|}{ Modes } & \multicolumn{2}{c|}{ Modes } & \multicolumn{2}{c|}{ Modes } \\
\cline { 2 - 7 } & $\mathbf{1}$ & $\mathbf{2}$ & $\mathbf{1}$ & $\mathbf{2}$ & $\mathbf{1}$ & $\mathbf{2}$ \\
\hline \multirow{2}{*}{ GMLS-Linear } & 12.36 & 482.3 & 496.4 & 3804 & 97.43 & 1561 \\
\cline { 2 - 7 } RB-Linear & 12.36 & 484.4 & 499.7 & 3776 & 97.25 & 1549 \\
\hline \multirow{2}{*}{ Ref. 14 } & 12.36 & 485.5 & 500.6 & 3805 & 97.41 & 1559 \\
\hline
\end{tabular}

\section{Euler Buckling:}

The Euler buckling load of a fixed-free column is evaluated by setting $\omega=0$ in Eq. (15).

Table 4 compares the non-dimensional buckling loads. Once again, both GMLS-Linear and RB-Linear methods gave Euler buckling loads.

Table 4: Non-dimensional Euler buckling load, $\left(P L^{2} / E I\right)$, for a fixed-free column 


\begin{tabular}{|c|c|c|}
\hline $\begin{array}{c}\text { N-Node } \\
\text { Model }\end{array}$ & GMLS-Linear & RB-Linear \\
\hline \multirow{2}{*}{5} & 2.48 & 2.453 \\
\cline { 2 - 3 } 17 & 2.467 & 2.464 \\
\cline { 2 - 3 } & 2.469 & 2.467 \\
\cline { 2 - 3 } 33 & 2.466 & 2.467 \\
\hline Ref. 14 & 2.467 & 2.467 \\
\hline
\end{tabular}

\section{Column with follower forces (Beck's Column ${ }^{10}$ ):}

A column with follower forces, (i. e. with $\gamma=1$ ) is analyzed next. The problem is known as the Beck's problem and is a problem with non-conservative loads ${ }^{10-13}$. While the Euler buckling can be analyzed by static methods, the non-conservative problems need dynamic stability criteria. The dynamic criteria for stability utilize disturbances produced by small vibrations on columns and interrogating the subsequent vibrations. If the vibrations die out, then the column is stable and if not the column is unstable (see Timoshenko and Gere ${ }^{12}$ pages $152-153$ for a detailed discussion on static and dynamic stability criteria).

The problem was analyzed by both GMLS-Linear and RB-Linear methods and four N-node models. Table 5 compares the critical load evaluated by the present methods and those from the literature. Excellent agreement is observed between the calculated critical loads and those from the literature ${ }^{10-12}$. The results obtained using the dynamic stability criterion with the 17-node model are presented in Figure 6. Both GMLS and RB models results are extremely close to each other and hence are indistinguishable on this figure. Figure 6 shows variation of the nondimensional frequencies for the first and second modes for various values of the nondimensional buckling load. For a given magnitude of the load on the column, the corresponding eigenvalues, $\omega$, are evaluated using Eq. (15). As the load is increased, the first two frequencies of the column approach each other, and at the critical load, the first and the second frequencies coalesce. Any further increase in load beyond this point leads to complex eigenvalues, suggesting that the column has reached into the unstable region ${ }^{10-13}$. 
Table 5: Comparions for critical loads, $\left(\mathrm{PL}^{2} / \mathrm{EI}\right)$, for Beck's column

\begin{tabular}{|c|c|c|}
\hline N-Node Model & GMLS-Linear & RB-Linear \\
\hline \multirow{2}{*}{$\mathbf{5}$} & 20.320 & 19.205 \\
\cline { 2 - 3 } $\mathbf{1 7}$ & 20.150 & 19.810 \\
\cline { 2 - 3 } & 20.042 & 19.989 \\
\cline { 2 - 3 } 33 & 20.108 & 20.035 \\
\hline Ref. 10-12 & $\mathbf{2 0 . 0 5 0}$ & $\mathbf{2 0 . 0 5 0}$ \\
\hline
\end{tabular}

\section{Concluding Remarks}

Two meshless local Petrov-Galerkin (MLPG) methods based on two different trial functions but using the same simple linear test functions were developed for beam and column problems. These methods used generalized moving least squares (GMLS) and radial basis (RB) trial functions. These two methods were tested on several patch test problems. Both methods passed the patch tests successfully. Then the methods were applied to various beam problems and Euler and Beck's column problems. Both methods yielded accurate solutions for all problems studied.

The simple linear test function offers considerable savings in computing effort as the domain integrals involved in the weak form are avoided. The methods produced accurate results for frequencies and buckling loads. Of the two methods studied, the GMLS-Linear method suffers from the drawback that the fictitious nodal values of the interpolation function do not pass through the actual nodal values (i.e does not satisfy the Kronecker delta property). Hence additional processes need to be implemented to prescribe the essential boundary conditions. In contrast, the method with radial basis trial functions does not suffer from this drawback. The implementation of essential boundary conditions is easy and straightforward. The method based on radial basis functions is robust and gives accurate results. 


\section{References}

1 Nayroles, B., Touzot, G., and Villon, P., "Generalizing the finite element method: diffuse approximation and diffuse elements," Computational Mechanics, Vol. 10, 1992, pp. 307-318.

2 Belytschko, T., Lu, Y. Y., and Gu, L., "Element-free Galerkin methods", International Journal of Numerical Methods in Engineering, Vol. 37, 1994, pp. 229-256.

3 Atluri, S. N. and Shen, S., The Meshless Local Petrov-Galerkin (MLPG) Method, Tech Science Press, Encino, CA, 2002.

4 Atluri, S. N., Cho, J. Y., and Kim, H. -G., "Analysis of thin beams, using the meshless local Petrov-Galerkin method, with generalized moving least squares interpolations," Computational Mechanics, Vol. 24, 1999, pp. 334-347.

5 Raju, I. S. and Phillips, D. R., "Further developments in the MLPG method for beam problems," CMES: Computer Modeling in Engineering \& Sciences, Vol. 4, No. 1, 2003, pp. 141-159.

6 Raju, I. S., Phillips, D. R., and Krishnamurthy, T., "A Radial basis function approach in the meshless local Petrov-Galerkin Method for Euler-Bernoulli beam problems", Computational Mechanics, Vol. 34, 2004, pp. 464-474.

7 Cook, R. D., Malkus, D. S., and Plesha, M. E., Concepts and Applications of Finite Element Analysis, Third Edition, John Wiley \& Sons, 1989.

8 Zienkiewicz, O. C. and Taylor, R. L., The Finite Element Method, Vols. 1 and 2, Fourth Edition, McGraw Hill Publishers, 1989.

9 Atluri, S. N., Kim, H.-G., and Cho, J. Y., " A critical assessment of the truly meshless local Petrov-Galerkin (MLPG) and local boundary integral equation (LBIE) methods", Computational Mechanics, Vol. 24, 1999, pp. 348-372.

10 Beck, M., "Die Knicklast des einseiting eingespannten, tangential gedrucken Stabes", Zeitschrift fuer Angewandte Mathematik und Physik, Vol. 3, pp. 225-228, 1952.

11 Leipholz, H., Stability Theory, Academic Press, New York, 1970.

12 Timoshenko, S. P. and Gere, J. M. , Theory of Elastic Stability, McGraw Hill, Second Edition, 1963.

13 Raju, I. S., "Analysis of a column subjected to follower forces by a meshless local PetrovGalerkin method", Paper presented at the $45^{\text {th }}$ AIAA/ASME/ASCE/AHS/ASC Structures, Structural Dynamics, and Materials Conference, Palm Springs, CA, April 19-24, 2004, AIAA 2004-1769. 
14 Weaver, W. Jr., Timoshenko, S. P., and Young, D. H., Vibration Problems in Engineering, John Wiley \& Sons, 1990.

15 Press, W. H., Flannery, B. P., Teukolsky, S. A., and Vetterling, W. T., Numerical Recipes The Art of Scientific Computing (FORTRAN Version), Cambridge University Press, New York, 1989.
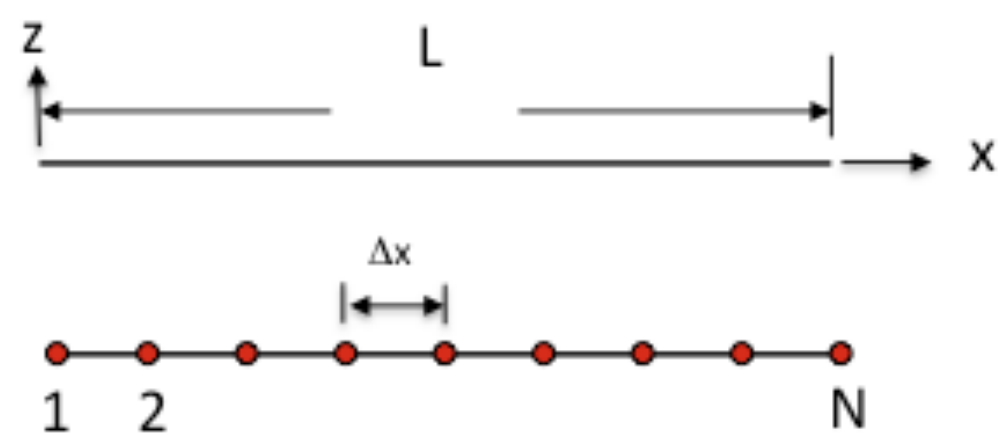

Figure 1. A beam and its $\mathrm{N}$-node meshless model

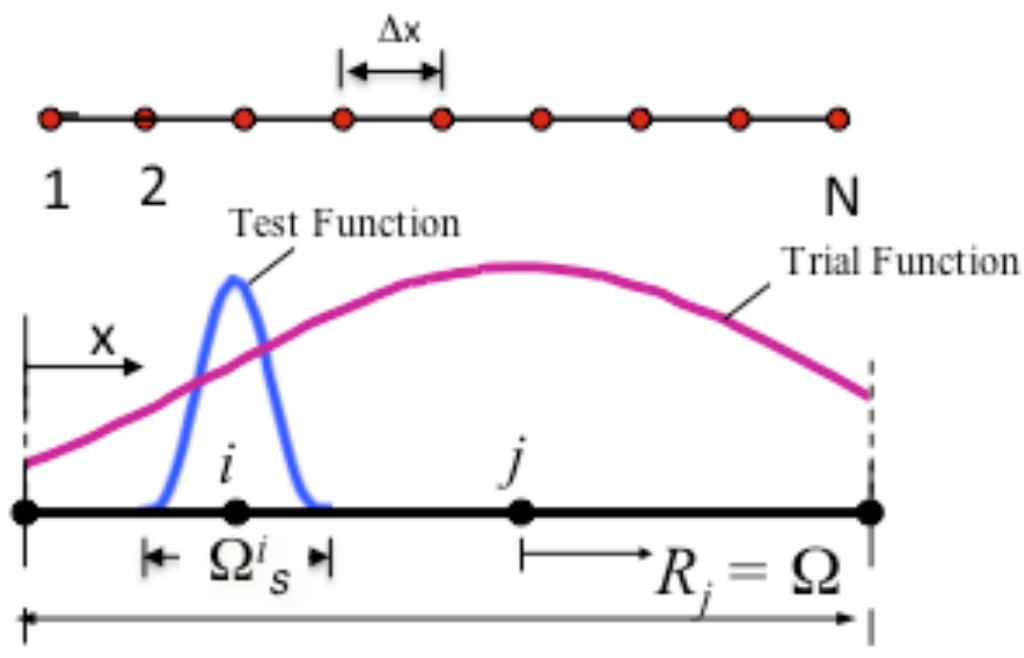

Figure 2. Trail and Test functions 
Fictitious nodal values

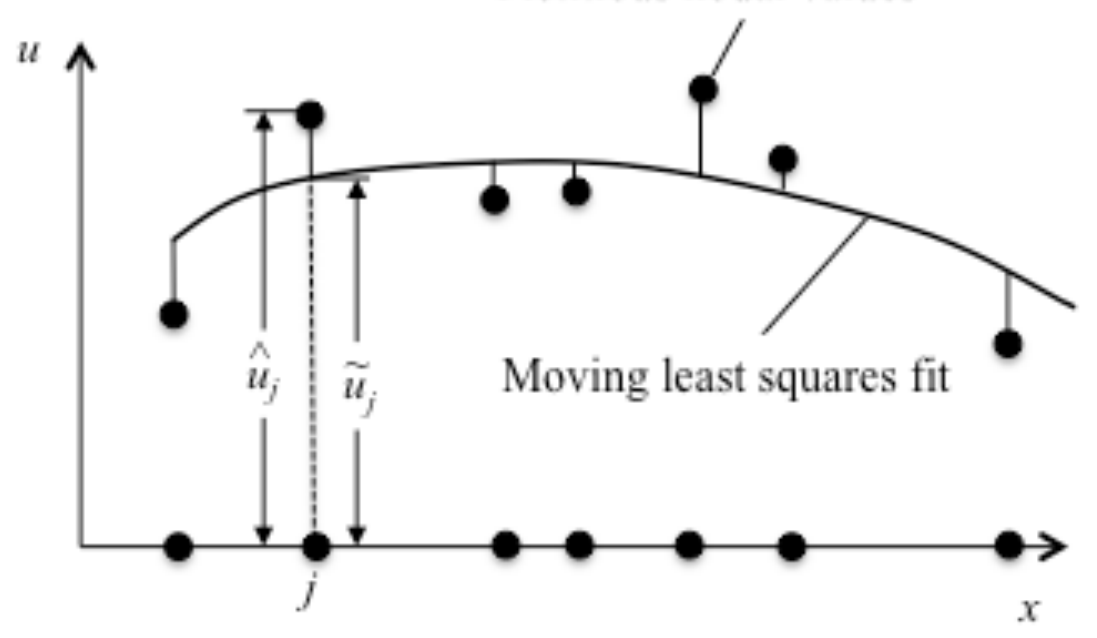

Figure 3. MLS trial function ( $\tilde{u}_{j}$-actual nodal values and $\hat{u}_{j}$ - fictitious nodal values)

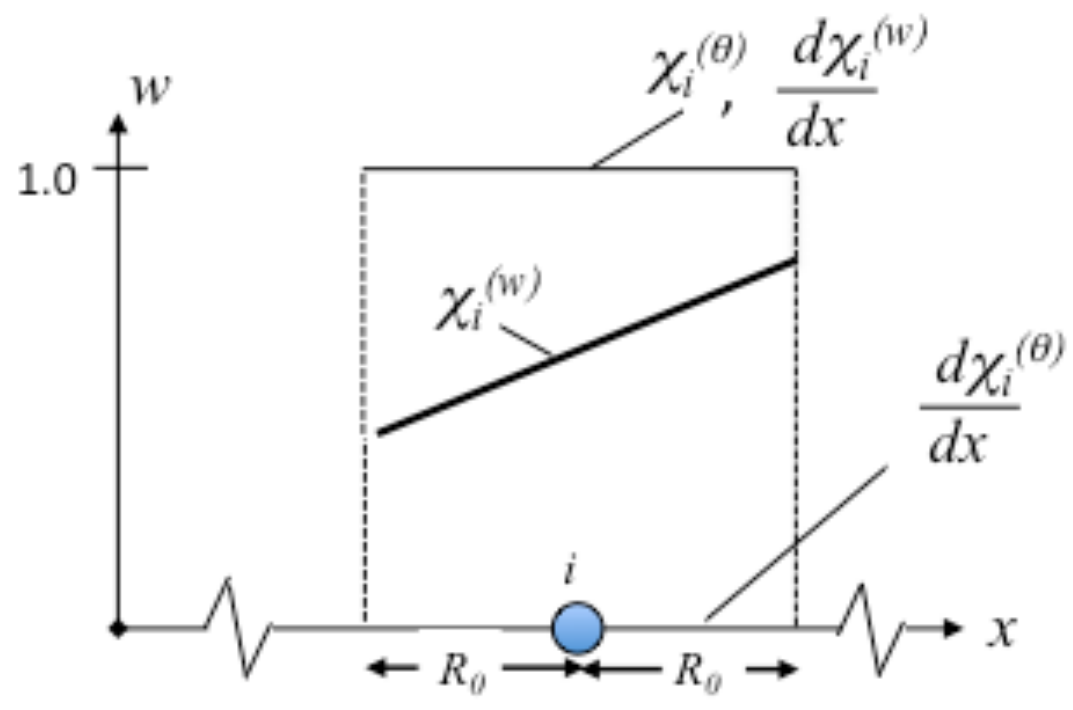

Figure 4. Linear test function at node $i$ 


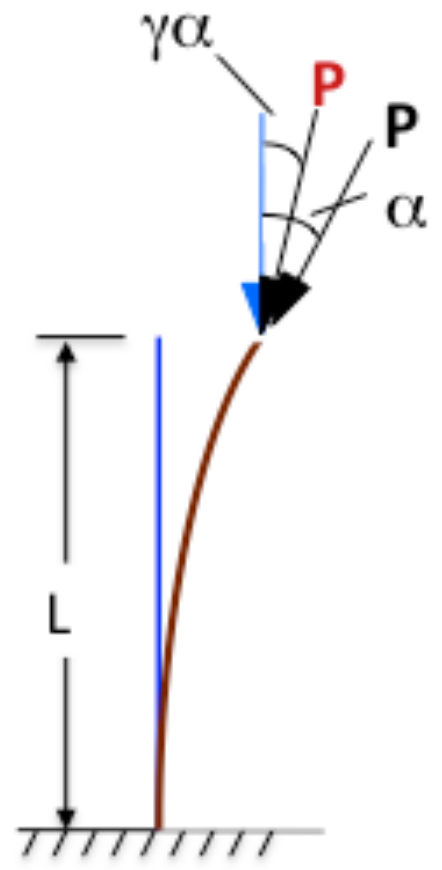

Figure 5. Buckling of a Column 


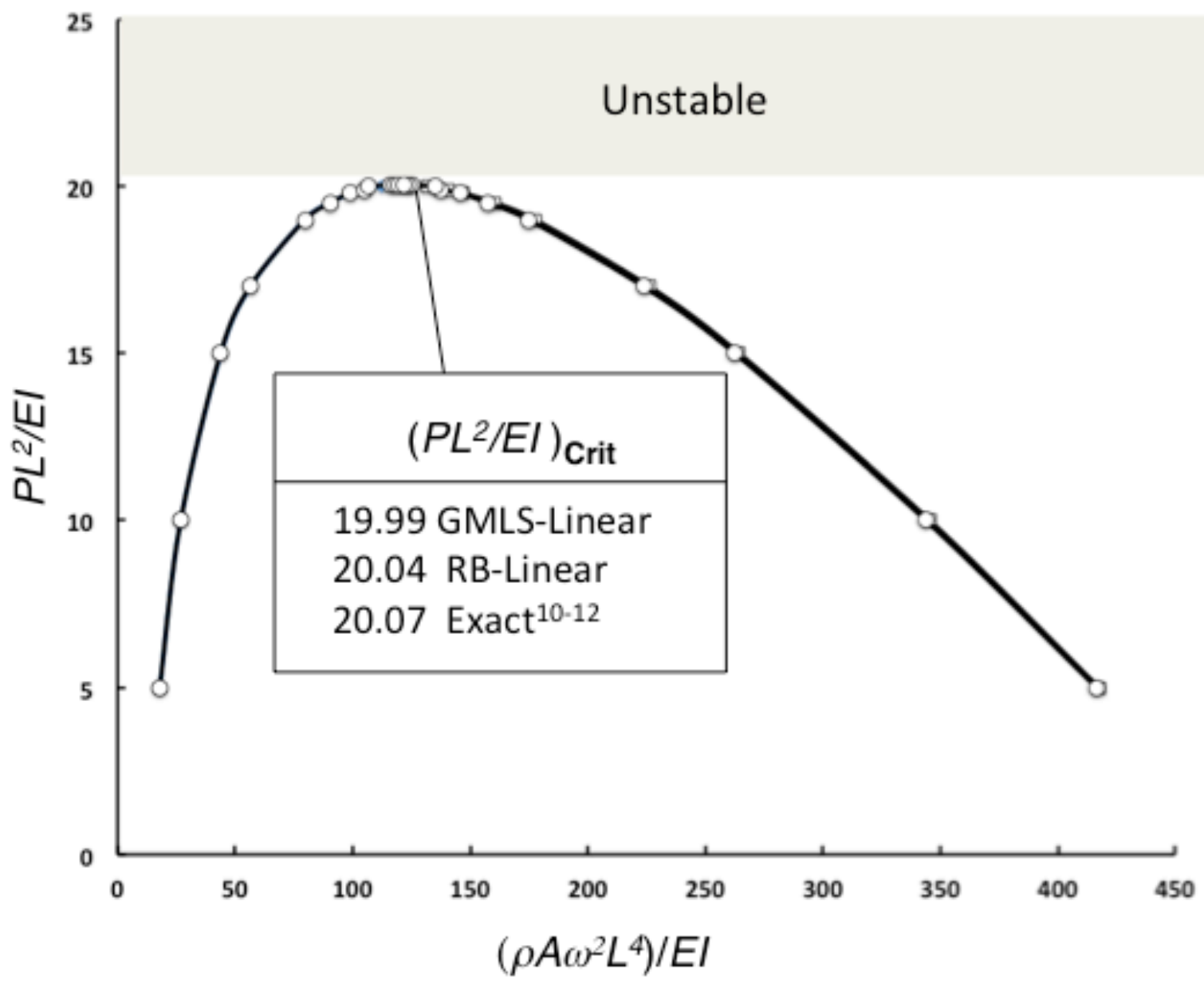

Figure 6. Eigenvalue curve for $\gamma=1$ (Beck's problem). 